\title{
DESIGNING ADAPTIVE ARCHITECTURES FOR TRANSOCEANIC IN- FLIGHT COMMUNICATIONS
}

\author{
Richard Slywczak, Okechukwu Mezu, NASA Glenn Research Center, Cleveland, Ohio \\ Bryan Green, Tennessee State University, Nashville, Tennessee
}

\begin{abstract}
During commercial flights, pilots require continuous communications and seamless access to data products, such as graphical weather maps and turbulence alerts, to proactively react to dynamic flight conditions. NASA/Glenn Research Center (GRC) and the Weather Information Communications (WINCOMM) project have been researching methods to improve communications and to disseminate graphical weather data products to aircraft flying in the transoceanic region where en route weather collection and dissemination are minimal. The goal is to employ commercial satellite-based communications and packet switching technologies to provide a cost effective and efficient communications solution for aviation.

This paper will describe the goals of the WINCOMM program and the research related to the transoceanic scenario. It will describe the flight architecture and the proposed communication network that is currently being implemented in the laboratory. The main goal is to have a seamless but efficient separation of services between the cockpit and cabin data with both data existing on the same data link. The initial findings for the Quality of Service $(\mathrm{Q} O S)$ research will be presented along with techniques for implementing QoS in Cisco routers and the design of the QoS schemes for the transoceanic testbed.
\end{abstract}

Data for the testing will initially focus on sending informational and graphical weather data but will eventually encompass warning/cockpit alerts and, hopefully, air traffic control messages. In mid-2005, the laboratory setting will be flight tested aboard the Langley Research Center's (LaRC) Boeing-757.

\section{Introduction}

The goal of the WINCOMM Project [1] at GRC, an element of the Weather Accident Prevention (WxAP) Project [2], is to develop advanced communications and information technologies to enable efficient and timely dissemination of weather information from the ground to the flight deck and turbulence information between aircraft. One of the scenarios currently being studied is the transoceanic region that deals with international flight operations over the oceans where communications are limited to the High Frequency (HF) spectrum and the en-route weather information collection and dissemination are minimal. To improve the timely dissemination of information to the cockpit, the research will focus on using satellite-based communications over regions without ground links, while being able to efficiently and automatically switch to ground links when they are available.

Currently, communicating with the cockpit is via a circuit-switching network. While circuit switching does provide a dedicated communications path with an allocated bandwidth, it does not make the most efficient use of the bandwidth or data links for small packets or short communication durations that could be achieved using packet-mode service. The existing aircraft data links are useful for transporting small, low volume messages, but they do not meet the needs of modern data products, such as graphical weather information. Future links will target large, high volume, and variable length messages.

The focus of the transoceanic scenario is to investigate the advantages of converting from circuit to packet switched mode using the INMARSAT/Swift-64 [3] service. Packet mode service provides a number of benefits, such as providing more efficient use of datalinks and bandwidth, and providing integrity and reliability for the data transmission. Furthermore, a costs saving can be realized by utilizing commercial services to provide the infrastructure. On-board the aircraft, the HSD-128 (High Speed Data) Unit will serve as the receiver and, currently, provides Internet connectivity to the cabin passengers for

\section{U.S. Government work not protected by U.S. Copyright}


services such as e-mail, web surfing, and video conferencing.

The issues for this research project will focus on the efficient separation of cabin and cockpit communications. The major issues will be quality of service, link availability and security. For instance, if a passenger was downloading a large image, the download process should not preclude the pilot from receiving important weather information about approaching storms or turbulence. The messages that are destined for the cockpit need to have a higher quality of service (or higher priority) that those destined for the cabin. The communications path between the aircraft and ground should always be available for data being transmitted; data should always be able to get to the crew of the aircraft. Finally, data must be transmitted to the cockpit securely and reliably. It is important for data to be transmitted quickly, reliably, and securely.

This paper will describe the goals and design approaches for the transoceanic scenario. The research will investigate and design an efficient onboard network that will provide services to crew and passengers during flight and the WINCOMM project will setup a laboratory environment to test this architecture under different scenarios. Given the limited number of passengers aboard the aircraft, the network must adapt to the users of that particular flight. The initial research emphasis is focused on QoS techniques that will prioritize data. Data for the testing will initially focus on informational and graphical weather data products and will eventually encompass warning/cockpit alerts and, hopefully, air traffic control messages. In mid-2005, the laboratory setting will be flight tested aboard the Langley Research Center (LaRC) Boeing-757. In addition, the researchers will investigate the new Inmarsat-4 satellites that launch in the 2004/2005 timeframe and, not only to analyze the Swift-64 service, but also examine some of the newer services.

As a result of this research, data communications from ground-to-air and air-to-air can be greatly improved and higher-quality data products, such as graphical weather products, can be quickly and more efficiently transmitted to the cockpit.

\section{Transoceanic Architecture}

The overall communications architecture, as shown in Figure 1, describes the transoceanic flight region. The problem can be divided into two segments. First, the communications architecture describes the communications patterns involving the aircraft, ground controllers, and satellite equipment. All data will be sent via either public or private networks to a commercially owned ground stations and sent to the appropriate destination through the Inmarsat/Swift-64 communications service. The commercial provide for this service is SITA [4]. Second, the on-board networking architecture will describe how the data will be routed on the aircraft to provide efficient service to both the cabin and cockpit of the aircraft. The onboard network architecture will be described in a following section.

The transoceanic communications architecture will consist of three types of communication patterns [5]:

- Ground-to-Air: The ground-to-air segment is initiated by a ground component and will transfer data products to a specified aircraft. The initiator could be air traffic control (ATC) or dispatch that will transfer the data over a private network segment or a commercial weather service that will transfer data over the public Internet. Both types of data will flow to a ground station that will broadcast the data via the Inmarsat/Swift-64 service to the aircraft. The types of data transferred over this link will be reports, alerts, warning or flight information from either ATC or the dispatch center. It could also be weather alerts or data products from a commercial provider.

- Air-to-Ground: The air-to-ground segment is the reverse of the ground-to-air segment where communications will be initiated by the aircraft and either makes a request or transfers data products to a ground segment. Depending on the destination, the data products could be transferred over either a private network or the public Internet. The types of data transferred over the air-toground link are the following: sensor or telemetry data that was measured by the aircraft and in situ weather data, such as 
turbulence, that can be transferred to the ground and can be broadcast to other aircraft flying in the same region.

- Air-to-Air: The air-to-air link will permit on-board sensed data, such as turbulence warnings and alerts, to be transferred to other aircraft flying in the same region. The definition of an air-to-air transfer in the WINCOMM project will include a hop through a ground terminal. So, if an aircraft sensed turbulence, it must first transfer that data to the ground and then it will be broadcasted from the ground terminal to all aircraft in the area.

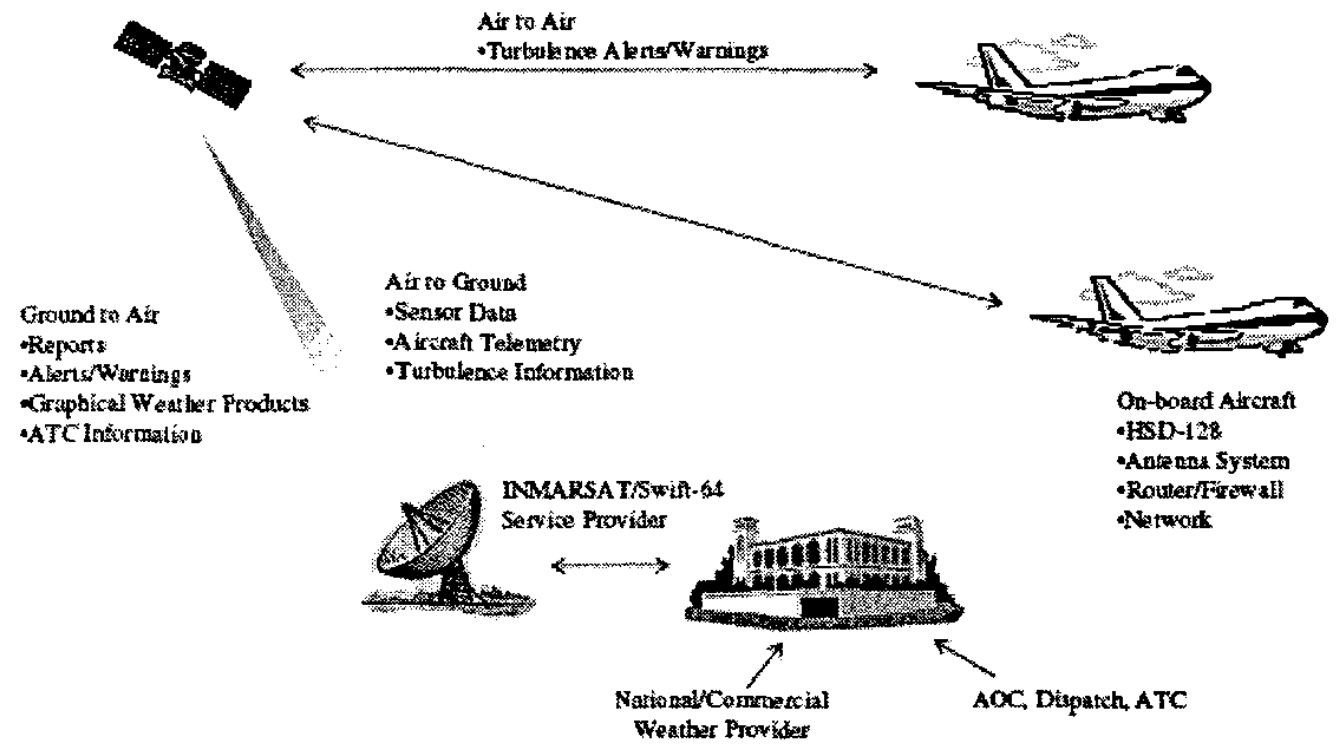

Figure 1. Transoceanic Architecture

\section{Program Overview}

Given that the focus of the WINCOMM project is on aviation safety and weather related research, the program goals for the transoceanic scenario focus on providing the pilots of transport with the best weather information in the timeliest manner. The main products will be turbulence detection and graphical weather products. The project will expand to include cockpit warnings/alerts and, hopefully, ATC data. The goals, as listed below, serve as the high-level users requirements [6]:

- On-board sensed turbulence information to ground users and between aircraft (airground, air-ground-air).

- Broadcast graphical weather products to pilot (ground-air).

- Transmit cockpit warnings/alerts to the aircraft (ground-air).
- Transmit aircraft traffic control information to the aircraft (ground-air).

Each of these tasks can be further divided into the following subtasks that will be the focus of the transoceanic research project:

- Dissemination of data from own ship turbulence to other aircraft and ground users.

- Receive, process and deliver valid turbulence warnings to the cockpit from other equipped aircraft.

- Receive and display Flight Information Service Broadcast (FIS-B) ground-air weather products.

- Receive, process, and display cockpit warnings and alerts from the ground stations on-board the aircraft. 
- Receive and process ATC information from the ground stations to the aircraft.

- Verify that ATC information can be transferred reliably and securely using encryption and reliable protocols.

\section{Benefits of the Research}

There are a number of benefits that will directly result from this research effort. Since IPbased protocols ${ }^{1}[7]$ are already delivering content to the passenger cabin, the aviation community has already realized some of the advantages of putting data over a packet switched network. These same benefits can be extended to cockpit communications and summarized as follows:

- Reduce Costs - One of the most provoking reasons to switch to a packet switched solution (i.e., IP-based protocols) is reduced costs. These reductions can be realized in two ways. The first is to use commercial services rather than services tailored specifically to the aviation community. Since the commercial systems have a large user base and competition, they should be able to provide more flexible and efficient pricing plans. The second method of reducing costs is by taking advantage of the large body of research by the Internet research community. While IP-based protocols have been studied extensively, the research community is still finding methods of improving the protocols. By capitalizing on this research, the aviation community will have a standards-based protocol rather than having to maintain the infrastructure of a custom protocol.

- Efficient Bandwidth Usage - Currently, when data is being transmitted to the aircraft, circuit switching reserves a link and allocates the entire bandwidth. However, the bandwidth is not constantly used since communications is sporadic. In a packet switching mode, data are contained in individual packets that are sent over the network which provides an opportunity for multiple users to efficiently share the same

\footnotetext{
${ }^{1}$ IP-based protocols refer to the IP protocol suite which includes the TCP/IP and UDP protocols.
}

datalink. Since each packet is uniquely addressed, IP-based protocols simplify using the same data link for multiple users. Sharing the datalink makes more efficient use of the bandwidth.

- Data Integrity and Reliability - IP-based protocols have two built-in mechanisms that would be very important to the aviation community. First, IP-based protocols use checksums ${ }^{2}$ to ensure that the data is validated against tampering or corruption, prior to arriving at the destination. Research studies [8] have shown that a 16-bit checksum might not be adequate for critical data (e.g., ATC). So, for critically important data, a more elaborate checksum might be considered. In addition, IP-based protocols provide both reliable and unreliable communications. Reliability will ensure that the data has reached its destination and, if it hasn't, it can be retransmitted. For example, reliability would be more important in ATC or Alerts/Warning than in weather data.

- Security - In the terrestrial Internet, there has been a significant amount of research on the issue of security. Both IPSec [9] extensions to the standard IP-based protocols and Virtual Private Networks (VPN) [10] are being implemented to provide secure communications. The aviation community has the opportunity to leverage these technologies to provide secure communications between the ground and cockpit. One significant advantage is that encryption can be implemented on the packet level, so communications with the cockpit can be encrypted while communications to the cabin is not. This provides the aviation community with the flexibility that it needs for secure but efficient communications.

\footnotetext{
${ }^{2}$ TCP has checksums enabled by default but, in UDP, checksums must be enabled by the programmer (i.e., they are not used by default).
} 


\section{Testbed Environment}

The function of the testbed environment is to test software and hardware in a laboratory setting that mimics the operational environment before flight testing. In addition, the testbed should help minimize the time needed for the actual flight testing. It will be used to test and verify the architectures, parameter modifications, and any software developed or changed during this research effort. It will also be able to communicate over the Inmarsat/Swift-64 Service and, therefore, the performance will be representative of what is expected during actual flight testing. Figure 2 shows an architecture in the testbed environment; the dotted lines represent advanced enhancements to the testbed and will be implemented, if time permits.

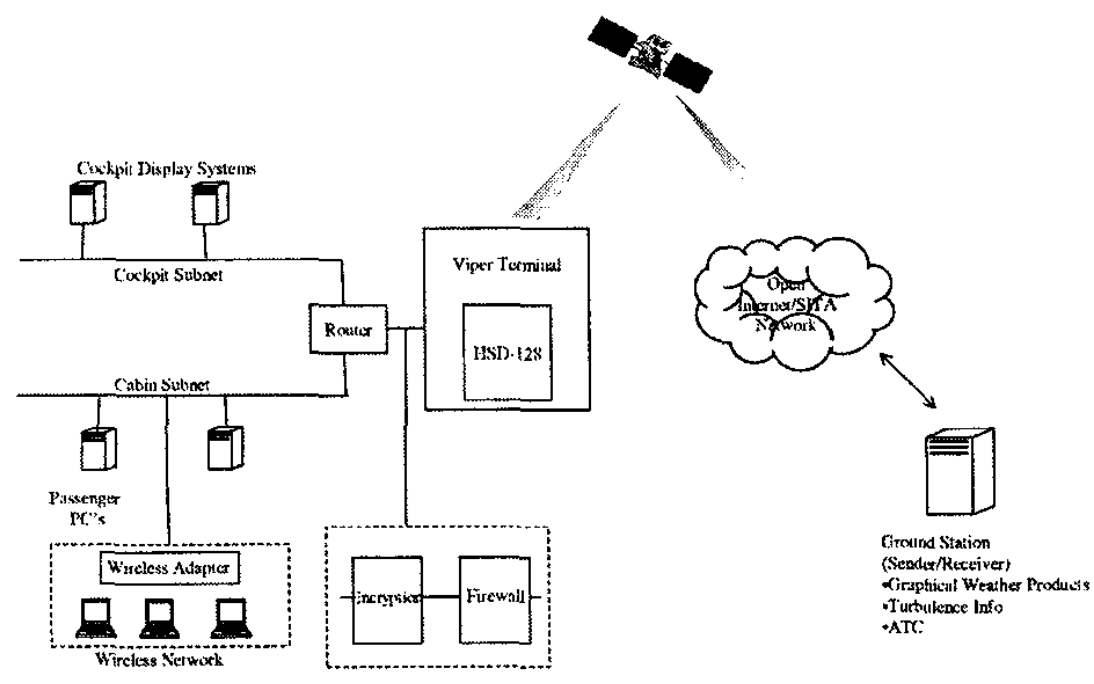

Figure 2. Testbed Environment

The testbed can be divided into two segments from the perspective of the Inmarsat satellite; the right segment represents the ground network and the left segment represents the equipment and networks on-board the aircraft.

On the right of the figure, the communications path is from a ground station (represented by a PC) through a public or private Internet. This could represent either the Internet or the private SITA network. The data is then transmitted to the satellite and sent to the appropriate receiver which, in this case, is the testbed.

The left side of the figure represents the network architecture that would be on-board the aircraft. The flow of data will come over the satellite and be received on-board the aircraft via the antenna - in the testbed, the antenna is roof mounted. The data will be received from the antenna by the High Speed Data (HSD-128) Unit which will output the data in packetized form. The
HSD-128 is a data transceiver for the Inmarsat/Swift-64 Service. It is capable of accepting two ISDN channels or a single Ethernet connection - our research will only consider the Ethernet capability. Each channel has a data rate of $64 \mathrm{~kb} / \mathrm{s}$ or the channels can be bonded together for a combined data rate of $128 \mathrm{~kb} / \mathrm{s}$. Using ISDN, both channels can be bonded together for data or they can be used independently for either data or voice service. With the Ethernet interface, only one channel will be active for a data rate of $64 \mathrm{~kb} / \mathrm{s}$ [11].

The packetized data that flows from the HSD128 to the router will be distributed on one of the two subnets, in this architecture. Dividing the network into subnets provides three significant advantages, as follows:

- Security. Using subnets, the router will be able to separate the data into cabin data and cockpit data. By doing so, the two types of 
data will not be intermixed on the same wire (i.e., the cockpit data will be on one subnet and the cabin data will be on another).

- Reduction of Data Traffic. Using two subnets will reduce the amount of data that will have to transverse each segment. This will help to reduce the number of data collisions and improve the efficiency of the network.

- Quality of Service. Another added benefit of multiple subnets is that they should provide an advantage with the Quality of Service issues. Once the data arrives on-board the aircraft, it can be classified as either cabin or cockpit data and can be immediately prioritized to the appropriate network.

Before flight testing, additional enhancements to the testbed will be as follows: addition of a wireless segment on the subnet so that cabin passengers can bring laptops on board and access the network; addition of a firewall to scan the data for basic security checks; addition of encryptors to encrypt command and control data that is destined for the cockpit; and testing a Virtual Private Network (VPN).

\section{Network Design}

The on-board networking must be designed to change or adapt to the situation and the individuals that are on a particular flight. Unlike a terrestrial network where aspects of the network are fairly static, the on-board network will change based on people bringing new equipment on-board the aircraft or dynamically reconfiguring the equipment that is available for on-board use or preferences. Some of the aspects of the network that were designed to incorporate these dynamic qualities are:

- Self-Configuration. There will be a number of people on-board the flight for a short duration of time. They might have the option of bringing their own equipment (e.g., laptops) that will connect to the network or need to reconfigure any existing equipment provided by the airlines. The network includes a wireless segment to help accommodate this self-configuration.
- Security. With the number and quantity of people that will join and leave the network at anytime (as each flight ends and another begins), there must be adequate security measures to accommodate the traffic. In this network, we are going to test firewalls, VPNs and encryptors as mechanisms to protect the data flowing onto the aircraft.

\section{Quality of Service}

Being that the goal of this research effort is to provide both cabin and cockpit data over the same data link, Quality of Service (QoS) becomes an important issue, since the cockpit data should have a higher priority than the cabin data. QoS can be defined as a network's ability to provide consistent performance for a specified service. In this case, consistent performance refers to prioritizing the packets and the specified service refers to the packet delivery.

While the final network configuration is represented by Figure 2, the current configuration is represented by Figure 3. It represents the on-board segment which consists of a cockpit and cabin computer on their respective subnet and an end system that represents a computer at some end point on a public or private Internet. The end system will run a web server to provide simulated web sites and weather data. At this point, the satellite connection is represented by a serial cable that connects the two routers. At any time, the serial link can be replaced with the satellite link to provide real-world testing of the architecture. In this configuration, the routers are a 2501 (IOS 12.1) and a 2524 (IOS 11.1). Both routers are in the Cisco 2500 class. Each of the routers in the configuration is a Cisco 2500 running Internet Operating System (IOS) version 12.1. Our approach to implementing $Q o S$ in the architecture is to use and configure the schemes present in the IOS rather than attempting to develop custom software. When this activity goes from the research to design and implementation, being compatible with existing routers is paramount rather than attempting to populate new code for the QoS schemes to function. 


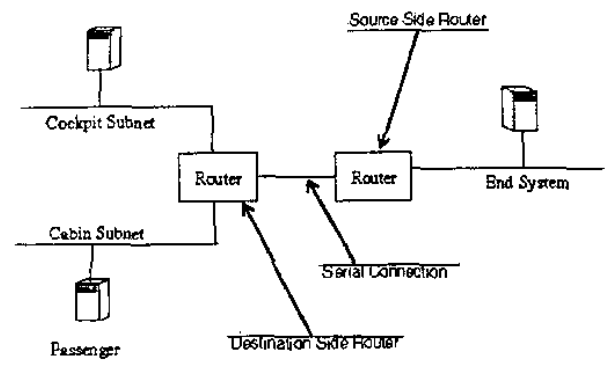

Figure 3. Initial Testbed Environment

Cisco IOS has four aspects to QoS which includes classification, marking, policing and shaping, and queuing. Classification consists of separating network traffic into a specified class of service. The separation of classes can be based on an incoming interface, a source or destination address, or an application. Marking consists of putting some type of mark on packets that enter or leave the router according to the classification scheme. Policing and shaping is a reaction to the marked packets. This aspect of QoS controls the traffic entering or leaving the router, so that the network conditions are predictable. Finally, queuing controls how and in what order packets are allowed to enter and leave the network [11].

\section{QoS Techniques}

Cisco provides a number of $\mathrm{Q} O S$ techniques contained in IOS; this section will provide a brief review of those techniques as follows:

- Policy Based Routing (PBR) - This mechanism forwards/routes data packets based on predefined polices according to Access Control Lists (ACLs) which are used to classify particular traffic by implementing a sequential list of permit and deny conditions. These lists can be applied to an interface on the router and used to filter data on an application, protocol, port number, and/or IP address. PBR also allows for the marking of the IP precedence bits located in the type of service field - the first three bits represent the IP precedence field -of the IP header. This technique was first introduced in IOS Software Release 11.1 [13].

- Priority Queuing (PQ) - PQ ensures that important traffic gets precedence over others. The network traffic using PQ can be prioritized by the network protocol, incoming interface, packet size, source/destination address, etc. Packets are categorized in one of four queues - high, medium, normal, or low - based on assigned priority. This technique was introduced in IOS Software Release 11.1 [14].

- Weighted Fair Queuing (WFQ) -WFQ separates traffic based on source and destination or MAC address, protocol, source and destination port, and socket numbers of a particular session. The classification scheme allows network traffic to be distributed in conversation like schemes based on fair queues. Data is placed in queues based on the time it takes for the last bit of each packet to arrive. WFQ was first implemented in IOS Software Release 11.1 [11].

- Class-Based Weighted Fair Queuing (CB$W F Q$ - CB-WFQ improves WFQ by allowing user defined classes of traffic. Traffic can be classified based on matching protocols, ACLs, and/or input interfaces. Bandwidth, weight, and maximum packet limit are applied to classes during congestion periods. CBWFQ was first implemented in IOS 12.1 [11].

- Committed Access Rate (CAR) -CAR performs two QoS functions. First, packet classification is performed by separating traffic based on port numbers, source or destination IP or MAC addresses, application ports, or the IP protocol type as specified in the ACL. After the data is classified, it is marked using the IP precedence bits. CAR also has the ability to police and shape data by managing the bandwidth for a given class of traffic through rate limiting. Traffic that falls between specified rates is transmitted while the other data is either dropped or placed in different priority categories. CAR represents one of the newer techniques and was implemented in IOS Release 12.1 [15].

- Weighted Random Early Detection (WRED) - WRED is designed to avoid congestion before it becomes an issue. It combines the Random Early Detection (RED) algorithm with IP precedence to 
provide preferential traffic handling for high priority packets. WRED can be configured to discard lower priority traffic when the interface begins to get congested. WRED was first implemented in Cisco IOS version $11.1[12]$

\section{QoS Design}

Based on the QoS schemes identified in the previous section, the testbed will be configured to prioritize packets based on their parameters. The implementation in the testbed will determine which scheme(s) are applicable and most beneficial to the transoceanic issues. There are two design methodologies used for implementing these schemes, as follows:

- Destination Router Implementation - The destination router implementation will attempt to distinguish between incoming packets based on the native TCP and IP packet header fields. Most importantly, this design does not imply setting any QoS bits on the source router side. Some of the inherent knowledge will be the destination IP or subnet addresses, port numbers, and/or protocol types. The types of $\mathrm{QoS}$ schemes that would be usable in the implementation would be Policy Based Routing and Priority Queuing. The benefit of detecting QoS on the destination side is that the source side entities, which could be a commercial weather service, would not have to adopt the QoS schemes or the packets would not have to be intercepted in the middle, such as before they are transferred to the satellite, to add QoS bits. This implementation is centrally located. The other major benefit is that the destination implementation provides a simplistic scheme that can be implemented easily. The disadvantage of this design is that QoS detection only on the destination creates a difficult problem since it relies on native packet information. In addition, a spoofed packet could set a high priority bit erroneously and, hence, permits sending rouge packets to the cockpit subnet. This design will have to implement security mechanisms (e.g., firewalls or encryption) to catch and/or eliminate the rogue packets.
- Source and Destination Router Implementation - The source and destination router implementation will have the source side (i.e., the router nearest to the source) set QoS bits in the TCP/IP header and have those bits interpreted by the destination. The sending side will maintain knowledge of the destination, since they will have opportunity to negotiate this information beforehand. The types of QoS schemes that would be usable in the implementation would be Weighted Fair Queuing, Class-Based Weighted Fair Queuing or Committed Access Rate. There are a couple benefits for this implementation. The first is that source and destination router implementation is more predictable than the destination only implementation. The source side will be able to easily set the QoS bits, since it bas knowledge of the packets and the destination will only have to interpret the QoS bits to determine the priority of the packet. Another advantage is that it provides a greater degree of security, since the packet will not only contain the IP address but also the QoS bits. It would be more difficult to spoof this information than just the IP information. The packet is also coming from a trusted source. The disadvantage of this implementation is that the processes are more complex, since it requires both the source and destination to implement the scheme. Agreements must be finalized beforehand, since some of the entities will involve commercial companies.

\section{QoS Classification Schemes}

Two important topics, regarding QoS, have been discussed. First, the types of QoS schemes contained in the Cisco router have been identified and the QoS design has been described. This section discusses the type of classification parameters that can be used with the schemes. The classification parameters provide a mechanism for filtering and prioritizing the packets as they pass through the router. These include both types of design where the destination router can classify packets based on the native packet header data or information that has been placed in the packet by 
the source router. The classifications parameters are as follows:

- Address Based Classification - On-board the aircraft, the user community is fairly small. One of the possible classification schemes would be to either prioritize based on the destination IP address or the subnet address of the packet. Based on the IP or subnet address, it is obvious whether the packet is destined for the cockpit or the cabin and, if the cockpit, which computer it will be received by.

- Data Type Classification - The second type of classification would be based on the type of data being received. For example, weather data and alert data would receive higher priority than generic web data. This classification could be combined with Address Based Classification and, once cockpit data has been isolated, they can be subcategorized and cockpit ATC or alert data would be higher priority than cockpit weather data.

- Predefined QoS Classification - Predefined QoS is simply QoS bits that have been previously set by the source router. While this seems like an obvious classification, it is important to recognize that this scheme might be used in conjunction with another, since all data might not be pre-classified before it reaches the destination router because source router QoS schemes could not be implemented in all necessary commercial routers. - this implies an assumption on where the implementation occurs.

- Quantity of Traffic - If the link is being over utilized by a single user, a QoS scheme could be implemented so that the traffic could be given a lower priority. This would assume a more fair utilization of the link.

\section{Future Work}

The transoceanic scenario is currently in the implementation phase. The initial testbed has been implemented and the QoS schemes have been studied and designed. However, there is still a significant amount of work left to be accomplished. Some of these issues are as follows:
- Continue the implementation of the QoS Schemes -The schemes that were described above will be implemented in the testbed and tested based on the following parameters: ease of implementation, performance and consistency in sorting and/or filtering packets.

- Implement Security Mechanisms - Security is becoming a major issue in networking research and is being considered as one of the major issues in this research effort. The security issues will include software solutions (e.g., firewalls) and hardware solutions (e.g., data encryptors). Each of these methods will be validated for security. concems and any impacts they have upon performance.

- Implementation of Advanced Technologies Once the basic issues of this study has been accomplished, the research will look at advanced technologies and how these might benefit the occupants of the aircraft. Some of these include the implementation of a wireless network on-board the aircraft so that users can attach to the Internet without physical plugging into a backbone and the implementation of IPv6 to avoid some of the addressing problem with IPv4.

- Flight Testing - In mid-June 2005, the testbed will be placed on the Boeing 757 research aircraft stationed at Langley Research Center (LaRC). Each of the QoS and security techniques will be tested and validated.

\section{Conclusion}

This paper proposes a method for improving communications during transoceanic flights. The improvements come in two phases. First, the current communications links are becoming outdated and would benefit significantly from newer technologies. By changing from circuit switched technology to packet switched technology and standards-based protocols, the aviation community could reduce costs and infrastructure while gaining integrity, reliability and security. While benefits can be realized, issues must first be resolved. Issues range from ensuring that important data gets to the correct destination in a timely manner, that the data link is always available and 
that security is maintained on the connection. To test these issues, we are in the process of constructing a testbed environment that will mimic the on-board setup. The testbed should provide a realistic communications environment by communicating directly with the Inmarsat satellite using the Swift-64 service. Our results will be verified during flight testing in mid-2005.

Secondly, the types of products going to the cockpit can also be greatly improved. Our research will focus on providing graphical weather products to the cockpit along with on-board sensed turbulence information that can be sent to the ground or to other aircraft in the area. These products will provide more detailed and real-time information to the pilots that help them avoid adverse weather conditions. Eventually, the research will encompass cockpit warning, alerts and ATC information.

The goal of this paper was to explain the project and document the QoS schemes that are currently involved in our research. We will continue to add functionality to the testbed that show the benefits of improved communications over the transoceanic flight regions.

\section{References}

[1] WINCOMM Project Homepage, 2004, Cleveland, $\mathrm{Oh}$, http://wincomm.grc.nasa.gov

[2] Weather Accident Prevention Homepage, 2004, Cleveland, Oh, http://wxap.grc.nasa.gov

[3] "Inmarsat - The Total Communications Network", 2004, http://www.inmarsat.org

[4] Sita Homepage, 2004, http://www.sita.aero

[5] Green, Walter, 2003, "Concept of Operations for the NASA Weather Accident Prevention (WxAP) Project", Hampton, VA, Langley Research Center, pp. 6-16

[6] Jarrell, Michael, 2004, "Preliminary Datalink Architectures for the Flight Demonstration of
Weather Dissemination Technologies", Cleveland, Ohio, Glenn Research Center, pp. 22-23

[7] Stevens, W. Richard, 1995, "TCP/IP Illustrated Volume I", Volume I, Upper Saddle River, NJ, Addision Wesley Publishing Company, pp. 223227.

[8] Stone, Jonathon, Craig Partridge, 2000, "When CRC and TCP Checksums Disagree", New York, NY, ACM Press (SIGCOMM 2000), pp. 1-11.

[9] Kent, Stephen, Randall Atkinson, 1998, "Security Architecture for the Internet Protocol", IETF RFC 2401, ftp://ftp.rfc-editor.org/innotes/rfc2401.txt

[10] Gleeson, Byran, Juha Heinanen, Arthur Lin, Grenville Armitage, Andrew Malis, 2000, "A

Framework for IP Based Virtual Private Networks", IETF RFC 2764, ftp://ftp.rfc-editor.org/innotes/rfc2764.txt

[11] EMS Technologies, Inc, 2003, "HSD HighSpeed-Data SATCOM Transceiver - Installation and Maintenance Manual", Revision A, Ottawa, Ontario, Canada, EMS Technologies Canada, Ltd., pp 104-131.

[12] Cisco Systems, 2003, "Internetworking Technology Handbook: Quality of Service", San Jose, CA., Cisco Press, pp. 49-3 - 49-18, http://www.cisco.com/univercd/cc/td/doc/cisintwk/i to doc/index.htm.

[13] Adams, Kevin, Spana George, Tan Nguyen, , "Router Support for Quality of Service", Virginia Tech., pp, 1-6, http://www.ee.vt.edu/ ldasilva/6504/router rep.doc

[14] Cisco Manual, 2003, "Congestion Management Overview", San Jose, CA, Cisco Press, pp. 36.

[15] Welcher, Peter, 2000, "New Quality of Service Features in Cisco IOS 12.1", Chespeake Netcraftsman, pp. 9, http://www.netcraftsman.net/welcher/papers/newqo s12.1.html 